\title{
Exploration of the Influence of Smiling on Initial Reactions Across Levels of Facial Attractiveness
}

\author{
Stephanie M. Shields*a, Caitlin E. Morse ${ }^{a}$, Paige Arrington ${ }^{b}$, and David F. Nichols \\ ${ }^{a}$ Department of Psychology, Roanoke College, Salem, VA \\ ${ }^{b}$ Graduate Program in Liberal Arts, Hollins University, Roanoke, $V A$
}

Students:smshields@mail.roanoke.edu*,cemorse@mail.roanoke.edu, arringtonap@hollins.edu Mentor:dnichols@roanoke.edu

\begin{abstract}
Both attractiveness and emotionality independently affect perception and interact to influence how a person perceives others. It has previously been shown that expressing positive emotions increases perceived attractiveness in general, but the relative influence of smiling across attractiveness levels and timing of this interaction is unknown. Such an interaction could entail dependent brain processing with interactions between brain areas or independent processing within each brain area. The present studies aimed to investigate this interaction and how it occurs through behavioral, specifically self-report, and physiological, specifically electrophysiological, methods. In each study, female undergraduate participants were shown images of male faces with smiling or neutral expressions. Study 1 used participant ratings to provide insight into the interaction and to establish an image subset of faces of high attractiveness (HA) and low attractiveness (LA). An interaction was found wherein HA faces were rated significantly higher on attractiveness when smiling whereas LA faces were rated similarly attractive regardless of emotional expression. Study 2 used electroencephalography (EEG) to examine the timing of brain responses to attractiveness, emotionality, and their interaction. Though a main effect of attractiveness consistently occurred prior to a main effect of emotional expression across two data sets, the presence of an interaction effect was inconsistent. There was some evidence for independent processing wherein the earliest brain responses are predominantly affected by attractiveness and are influenced by emotional expression, but dependent interactions between modular processing areas cannot be ruled out. Together, these results help to shed light on the interplay of attractiveness and emotionality though additional research could help to clarify the timing of the interaction on a neural level.
\end{abstract}

\author{
ABBREVIATIONS \\ HA - high attractiveness; MA - medium attractiveness; LA - low attractiveness; FERET - Facial Recognition Technology; ERP \\ - event-related potential; EPN - early posterior negativity

\section{KEYWORDS} \\ Attractiveness, Emotionality, Emotional Expression, Smiling, Electroencephalography, Event Related Potentials
}

\section{INTRODUCTION}

How one appears to others can be important as first impressions can have a significant and lasting influence on how people perceive others. ${ }^{1}$ Physical appearance and current emotional expression are readily apparent upon first meeting someone, making these important factors to consider in understanding the initial reactions of others. Attractiveness in particular has been shown to be a major factor that can have an impact on how one is perceived, especially in regards to the age of the respective person. ${ }^{2}$ Specifically, and regardless of gender, attractive people are perceived as more socially desirable and are believed to have more successful life outcomes. ${ }^{3}$ Raters who either know or do not know the people they are rating judge and treat attractive children and adults more positively than unattractive children and adults. ${ }^{4}$ Emotionality, the observable component of emotion, has also been shown to affect the perceptions of others. ${ }^{5}$ For example, impressions are impacted by smiling and the display of angry facial expressions. ${ }^{5}$ Regardless of the sex of both the observer and the person being observed, smiling people are perceived to be more likable and intelligent ${ }^{6}$ and more attractive. ${ }^{6,7}$ The apparent influence of emotional expression on judgements of attractiveness implies that there is an interaction at play between the neural processing of facial attractiveness and emotionality. Previous research shows that attractive faces activate the medial orbitofrontal cortex (OFC) and that responses in the medial OFC to attractive faces are enhanced when the face was smiling, suggesting that the medial OFC classifies attractiveness and smiling expressions as rewarding for observers. ${ }^{8}$

To better understand the neural processing of attractiveness and emotionality, electroencephalography (EEG) can be used to measure neural activity through event related potentials (ERPs) evoked by images that are high or low in particular qualities. Prior findings on the timing of differential responses to high and low attractiveness and of positive versus neutral emotional expressions suggest that attractiveness is processed faster than positive emotions. Specifically, differences in ERP signals recorded 
after the presentation of attractive vs. nonattractive images appear by at least $250 \mathrm{~ms}$ post-stimulus ${ }^{8}$ and possibly earlier when participants are required to make judgements of attractiveness. ${ }^{9}$ Differences between negative emotional expressions and neutral expressions also appear early, i.e., within $250 \mathrm{~ms}$, but differences between happy expressions versus neutral expressions appear about 100 ms later. ${ }^{10}$ However, conclusions on the relative timing of the neural processing are currently based on results from separate studies. To our knowledge, no studies have tested the relative timing of attractiveness and emotional processing using the same set of stimuli nor have any studies reported on the timing of their interaction. The current study is an attempt to fill this gap in the literature.

Behavioral investigations, such as those using self-reported ratings, can provide insight into the potential existence and nature of a neural interaction between attractiveness and emotionality. If an interaction effect does exist, the timing of it needs to be explored with an electrophysiological measure in order to determine how the brain combines information about these two influences on facial perception. Three possibilities for the timing of the interaction exist. If attractiveness and emotionality are processed sequentially but then combined in a dependent manner, an interaction effect would arise only after the occurrence of both main effects. Based on the timing discussed above, this would be about $450 \mathrm{~ms}$ or more after the stimulus. If they are processed in parallel but information dependent on both components is required before the interaction can take place, the interaction effect would be expected to occur no earlier than a main effect of emotion, thus about $350 \mathrm{~ms}$ or more after the stimulus. If facial expression has a direct, independent effect on attractiveness and if processing of emotional expression is not a prerequisite for the interaction, the interaction effect could happen before the main effect of emotionality and could be observed at the same time as or soon after the main effect of attractiveness. This would be approximately in the time window of $200 \mathrm{~ms}$ to $300 \mathrm{~ms}$ after the stimulus. This last possibility is most consistent with modular processing of facial attributes which would suggest that the processing of attractiveness and emotionality involve different parts of the brain that interact with one another for some judgments but not others ${ }^{11}$. Prior research has indicated that attractiveness is processed in the medial OFC ${ }^{8}$ and emotional expression is processed in the amygdala as well as the OFC. ${ }^{12}$

The current studies aimed to investigate the impact of facial attractiveness and emotionality, specifically smiling expressions, on the perception of others through behavioral and physiological measures. The behavioral measures are largely exploratory regarding any influence of attractiveness level on the effect that emotional expression has on ratings of facial attractiveness and perceived happiness. The physiological measures are in part confirmatory in that we hope to replicate prior research on the relative timing of neurological responses to facial attractiveness and emotional expression and in part exploratory because of the different possibilities discussed above regarding the timing of a neurological interaction effect between facial attractiveness and emotionality. Study 1 had female participants rate the attractiveness and happiness of a wide range of everyday male faces that contained images of each face with a neutral expression and with a smile. Study 2 extended these findings by investigating brain responses to the subset of images that were rated highest and lowest on attractiveness in order to localize in time the differences found among conditions in Study 1.

\section{STUDY ONE}

The ultimate goal of Study 1 was to evaluate the existence and nature of an interaction between attractiveness and emotionality in the perception of facial attractiveness. A preliminary step, and additional aim, was to establish a four-category set of face images that clearly varied in attractiveness, i.e., a high attractiveness and a low attractiveness set of images, but that were also different in emotional appearance, i.e., were perceived as happier when smiling versus with a neutral expression. Without clear differences in those dimensions, definitive comparisons could not be made across image categories. The images needed to be rated across enough participants to establish confidently that they would reliably be considered relatively attractive or unattractive and relatively more or less happy. This would allow them to be used in Study 2 with a separate set of participants who were not performing explicit ratings of the stimuli. It was expected that happiness ratings would be influenced by emotional expression across all attractiveness categories. Having a set of stimuli for which the attractiveness ratings change as a function of emotional expression more so in one attractiveness category than another would allow us to unambiguously determine whether an interaction effect in the neural response between attractiveness and happiness is primarily driven by attractiveness or emotionality processing. Stimuli used in studies on attractiveness have come from a wide array of sources, such as photographs taken by the researchers, ${ }^{13,14}$ images taken from the internet, ${ }^{15}$ or pictures from previously established databases of face images. ${ }^{7,9,16,17,18}$ While all image sets have their utilities in answering various questions, they are generally limited in how representative they are of the general population. We sought to utilize face images that had a broader characterization of the general population, including a range of ages and ethnic groups. Such an increased heterogeneity of faces may reveal effects that have previously been masked when researchers use only a narrow range of images. For instance, using only college-aged male faces would not allow us to determine if college-aged females respond differently to smiles of various-aged males, and we would not be able to draw broader conclusions about patterns in the perception of attractiveness and emotionality. Therefore, we decided not to limit the range of face images based on age or ethnicity and instead used images of individuals of a variety of ages and ethnicities. 


\section{METHODS}

\section{Participants}

A total of 29 female undergraduate students enrolled in psychology courses participated in this study. No further information about demographics was recorded. Students were primarily in introductory level psychology courses and were recruited from a participant pool which primarily consisted of young college-aged women of European descent. The study was conducted in accordance with the Roanoke College Institutional Review Board.

\section{Stimuli}

Two hundred black and white photographs of men were obtained from the FERET facial stimuli database from the Defense Advanced Research Products Agency. ${ }^{19}$ Photographs depict close-ups of faces displaying happy and neutral facial expressions with multiple images per person, some including different backgrounds and partial obstruction of the face. Images taken of individuals from an angle other than their front were excluded. Images were also excluded if the background of the image was anything other than plain white, if the individual was wearing accessories that obscured the face (e.g., hats or sunglasses), or if the individual's eyes were closed. To avoid biases in image selection, the first 60 photos of randomly ordered male faces exhibiting a smiling facial expression that met these criteria and images of the same individuals exhibiting a neutral facial expression were selected for use in the study. Though demographic information was not tied to the images, from visual inspection we believe that the stimuli consisted of individuals of European, African, Middle Eastern, and Asian descent and that the ages ranged from that of the average college student to that of senior citizens (see Figure 1 for representative stimuli).

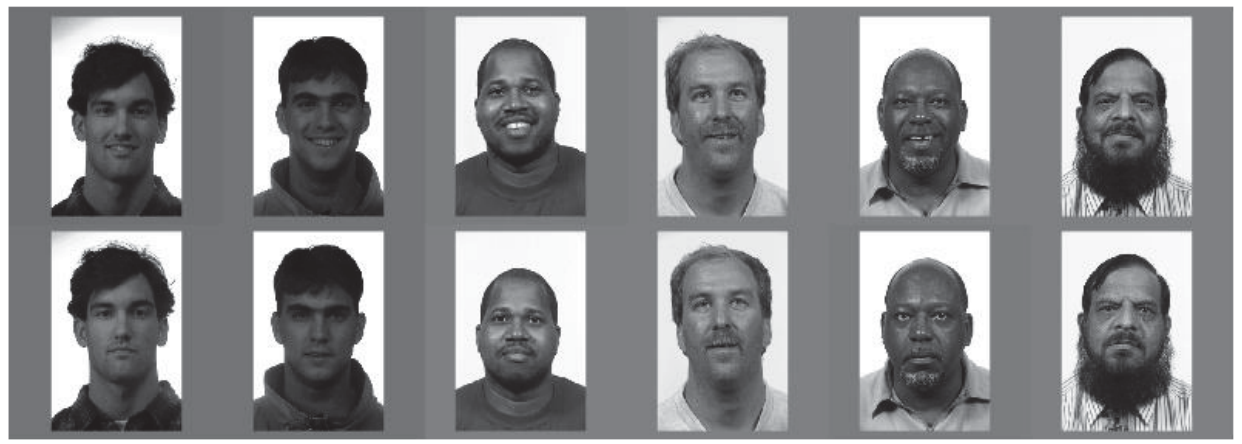

Figure 1. Example faces from the FERET face database used in Study 1. A set of two images were chosen per person, one with a smiling expression (top row) and one with a neutral expression (bottom row). Images varied in age, race, and ethnicity but were all of the opposite sex to the participant.

The face images were 384 pixels high and 256 pixels wide, embedded in a gray rectangle that was 420 pixels high and 420 pixels wide. The gray rectangle had an RGB value that was the midpoint of the face image RGB value (127) and was centered on a black screen $(\mathrm{RGB}=0)$ which was 900 pixels high by 1600 pixels wide. The rating scale was gray $(\mathrm{RGB}=150), 8$ pixels high by 730 pixels wide, and located 50 pixels below the stimulus image. The nine tick marks were 6 pixels wide by 16 pixels high and equally spaced along the rating scale, approximately 90 pixels apart. Text for the rating scale was gray $(\mathrm{RGB}=150)$ and centered below the corresponding tick marks.

\section{Equipment}

The computer used was a Mac Mini running Mac OS X version 10.6.8 connected to a 20" ViewSonic VX2033wm LCD monitor with a 1600x900 resolution at $60 \mathrm{~Hz}$. Stimuli were presented using MATLAB 2009b with Psychtoolbox-3 code. A chinrest was used to maintain a constant viewing distance of $30 \mathrm{~cm}$.

\section{Procedures}

Participants were tested individually in an isolated room. They were given a study information sheet to explain the fact that they would be completing two blocks and an instruction sheet with example images to explain the rating procedure before they began. In one block, participants were asked to rate the attractiveness level of the images on a nine-point scale from Very Unattractive (1) to Very Attractive (9) with intermediate points Somewhat Unattractive (3), Neutral (5), and Somewhat Attractive (7) also labeled. In a separate block, participants were asked to rate the level of happiness displayed in each photograph on a nine-point scale of Very Unhappy (1) to Very Happy (9) with intermediate points Somewhat Unhappy (3), Neutral (5), and Somewhat Happy (7) also labeled. Participants entered their rating for each image by moving the mouse cursor to the tick mark on the scale for their selection. 
The 120 images (60 smiling, 60 neutral expression) were displayed in a random order one image at a time. Images displaying smiling or neutral facial expressions were intermixed within a block, and therefore participants rated the same individual twice in each block. The order of the attractiveness and happiness rating blocks was randomized across participants. The face image and scale remained visible until a selection was made. Time expectancy for rating the images was approximately six minutes for each scale though participants were allowed to move at their own pace. The entire experiment, including initial training, took roughly 15 minutes per participant.

\section{Analysis}

Three different types of statistical tests were used to analyze separate aspects of the ratings data. The Wilcoxon paired sample signed-rank test was used for analysis of the distribution of individual ratings combined across all participants. A nonparametric test was chosen because the rating values are discrete rather than continuous. This test shows if attractiveness or happiness ratings are consistently higher for one emotional expression (i.e., smiling versus neutral). Correlations between the average ratings of each image across participants were also calculated. This test examines consistencies in attractiveness and happiness ratings across emotional expressions of the same individuals. Repeated Measures (RM) ANOVAs across attractiveness categories were conducted based on the average ratings across all images within each category. This test reveals if differences across categories are consistent across participants. A level of significance of $\alpha=0.05$ was used throughout.
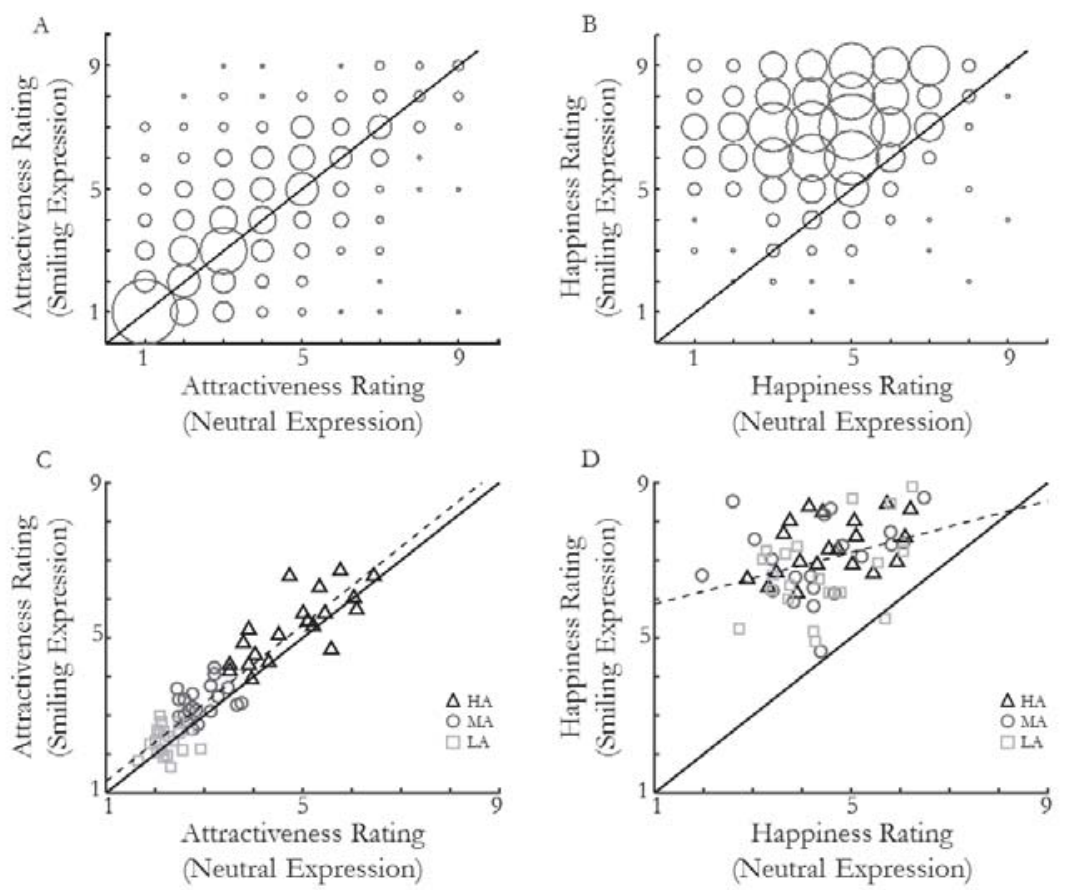

Figure 2. Scatterplots of the ratings across emotional expression for all ratings performed (A\&B) and on the basis of each of the 60 photographed individuals split into High Attractiveness (HA), Medium Attractiveness (MA), and Low Attractiveness (LA) categories (C\&D). Lines of equality are included as solid lines, and best fit lines are included as dashed lines. A) A bubble plot of the attractiveness ratings shows the relative frequency of particular combinations of ratings across emotion. Data are shown for all 1740 rating pairs obtained from the 29 participants rating 60 individuals each. B) A bubble plot of the 1740 happiness ratings across the same individuals as in A. C) A scatterplot of the attractiveness ratings of each of the 60 individuals averaged across all participants. D) A scatterplot of the happiness ratings of the 60 individuals averaged across all participants.

\section{RESULTS}

\section{Ratings}

Participants used a variety of attractiveness and happiness values when rating images (see Figure $2 \mathrm{~A} \& \mathrm{~B}$ ). In order to exclude clearly invalid responses, we made sure that no participant used only a single scale value in either rating block and that each participant consistently rated the smiling images as happier than the neutral images. No participants' data needed to be discarded. For the high end of the attractiveness scale, $52 \%$ of participants rated at least one image a 9, 94\% rated at least one image a 7 or above, and $100 \%$ used a rating of 5 or above at least once. This indicates that the image set included at least some images considered attractive by a vast majority of participants. Regarding the range of the attractiveness scale used by each participant, $45 \%$ used the entire width of the scale, $88 \%$ had a difference of at least 6 places from their highest to lowest ratings, and $100 \%$ 
had a difference of at least 2 places. For the high end of the happiness scale, $97 \%$ of participants rated at least one image a 9 and $100 \%$ used a rating of 8 or above at least once. Regarding the range of the happiness scale used by each participant, $66 \%$ used the entire width of the scale, $97 \%$ had a difference of at least 6 places for their highest to lowest ratings, and $100 \%$ had a difference of at least 5 places.

In order to determine attractiveness categories across all participants, the overall attractiveness rating for each individual depicted in the images was determined by averaging across emotional expression and the ratings of all participants (Figure 2C). The average attractiveness rating per individual ranged from 1.8 to 6.5. The 60 individuals were then rank-ordered based on overall attractiveness and split into three categories. The 20 individuals with the highest rating (from 3.7 to 6.5) were grouped into the high attractiveness (HA) category and the 20 individuals with the lowest rating (1.8 to 2.7) were grouped into the low attractiveness (LA) category. The remaining 20 individuals were grouped into the medium attractiveness (MA) category. The happiness ratings across images (see Figure 2D) were not used in the procedure to determine attractiveness categories.

Demographic information on the images was not included with the image set nor did the participants indicate what age and ethnicity they perceived the individuals in the images to be. However, possible differences in frequencies of different ages and ethnicities between the groups could be relevant for interpreting any observed differences between the different attractiveness categories. For each individual, the median age and most frequent ethnicity were determined across ratings made by the student authors. For the HA category, the mean age was 25, and age ratings ranged between 21 and 35 . Only one individual sorted in the HA category was perceived as not being of European descent. For the MA category, the mean age was 40.5, and age ratings ranged between 21 and 62. Four individuals in the MA category were perceived as not being of European descent. Finally, for the LA category, the mean age was 49.2, and age ratings ranged between 27 and 63 . As in the MA category, four individuals in the LA category were perceived as not being of European descent, so across the image set, nine individuals were rated as being of nonEuropean descent.

Consistency in Ratings Across Images

Participants demonstrated a large degree of individual differences in how attractive they perceived particular photographed individuals to be (see Figure 2A). However, a Wilcoxon signed-rank test across all 1740 ratings revealed a bias with individuals being rated as more attractive when smiling $(z=8.9, p<.001)$. There was much greater consistency for the happiness ratings $($ see Figure $2 \mathrm{~B}$ ) with a clear majority of the images being rated as happier when exhibiting a smiling expression compared to a neutral expression $(z=33.6, p<.001)$.

In order to examine the relative effects of emotional expression on the attractiveness and happiness ratings amongst photographed individuals, averages were taken across all participants for each individual separately. On the one hand, emotional expression tended to have a consistent effect on attractiveness rating across attractiveness levels (see Figure 2C), evidenced by the best fit line through the data having a slope of 1.0. The effect was relatively small with a vertical shift of 0.29 on a 9-point scale. On the other hand, emotional expression tended to have a large effect on happiness rating (see Figure 2D), evidenced by the best fit line through the data having a vertical shift of 5.53 on a 9-point scale and a slope of 0.33 . The average attractiveness rating, combined across emotional expressions, was not significantly correlated with the average happiness rating, $r(56)=0.22, p=.088$, indicating that ratings were likely based on distinct aspects of the images.

\section{Effects of Attractiveness Category}

As noted above, there was a large variation regarding which pictured individuals participants found attractive. Even within the set of images determined to be in the upper third of attractiveness (i.e., the HA category) and the lower third of attractiveness (i.e., the LA category) across all participants, across emotional expressions there was substantial variability between participants in the attractiveness ratings assigned to these individuals (see Figure 3A). For the HA category, there was a median rating of 5, and for the LA category, there was a median of 2 . There was also large variability in the happiness ratings for both attractiveness categories (see Figure 3B) though there was much more overlap in the distribution of ratings collapsing across emotional expression as the median for both HA and LA categories was 6.

Though individual differences in perceived attractiveness are inevitable, our goal was to have sufficiently consistent attractiveness ratings across the entire set of images within a category so that a different set of participants would experience the same differences between categories. The reliability of the effects of attractiveness category and emotional expression was therefore assessed across participants (see Figure 3C\&D). In order to determine the effects of attractiveness group and emotional expression separately and in combination on both the attractiveness and happiness rating scales, a 2x2 RM MANOVA was run. Regarding attractiveness ratings (Figure 3C), there was a main effect of attractiveness, $F(1,28)=119.6, p<.001, \eta^{2}=.780$, as would be expected based on the data categorization procedure. There was also a main effect of emotion, $F(1,28)=12.2, p=.002$, 
$\eta^{2}=.008$, though this was qualified by an interaction effect, $F(1,28)=12.4, p=.002, \eta^{2}=.003$. Further exploration using pairedsample $t$-tests demonstrated that smiling HA faces were consistently rated higher on attractiveness than neutral HA faces, $t(28)=$ $4.1, p<.001$, but smiling LA faces were not significantly different than neutral LA faces, $t(28)=1.3, p=.196$. Regarding happiness ratings (Figure 3D), there was a main effect of emotion, $F(1,28)=1032.5, p<.001, \eta^{2}=.924$ ), as would be expected based on the nature of the stimuli. There was additionally a main effect of attractiveness, $F(1,28)=114.8, p<.001, \eta^{2}=.027$, which was also qualified by an interaction effect, $F(1,28)=19.6, p<.001, \eta^{2}=.007$. Though smiling individuals were rated higher in happiness than individuals with a neutral expression for both the HA and LA categories $(t$ s $>26.6, p$ 's $<.001)$, the increase in happiness ratings was larger for the HA category than the LA category, $t(28)=4.40, p<.001$.
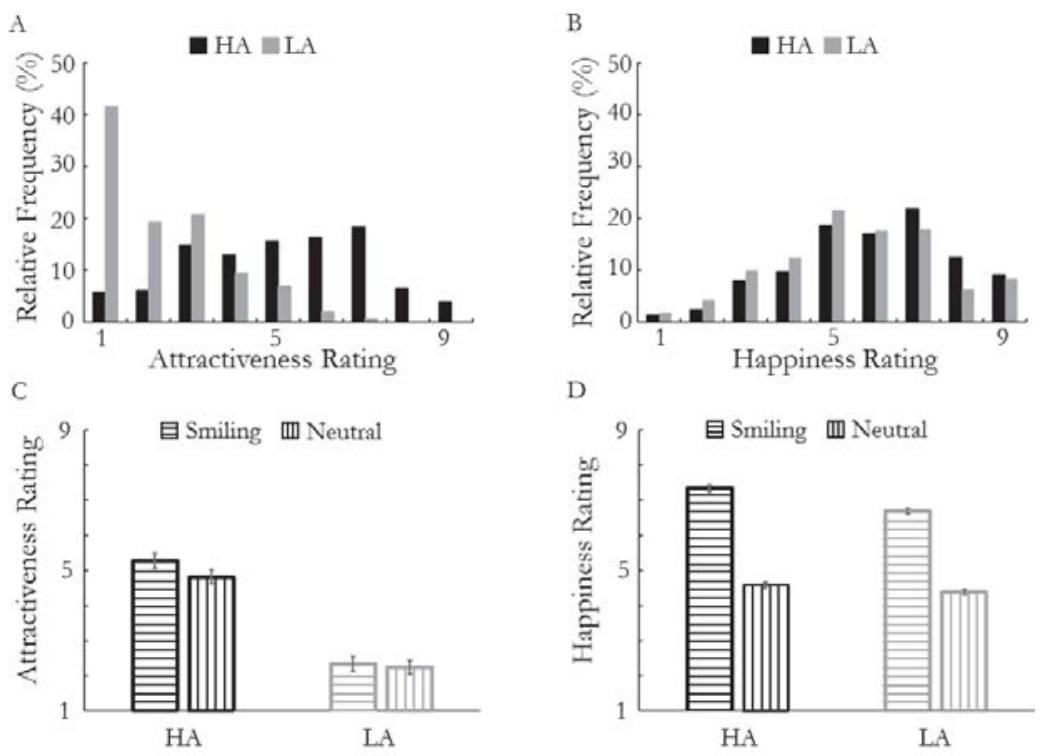

Figure 3. Effects of attractiveness category on the relative frequency of ratings used (A\&B) and cumulative averages across participants (C\&D). A) The relative frequency of attractiveness ratings across all images within the HA and LA categories. B) The relative frequency of happiness ratings across all images within the HA and LA categories. C) Attractiveness ratings averaged across all 20 images within a category as a function of the attractiveness level and emotional expression. D) Happiness ratings averaged across the same images as in C. Error bars show 1 standard error of the mean.

\section{DISCUSSION}

This study established image sets comprised of individuals of differing levels of attractiveness - HA and LA - displaying two different expressions - neutral and smiling. It was important that the image sets varied in attractiveness and perceived emotionality for the analysis of Study 1 data and so that the images could be used in Study 2 to examine brain responses during passive viewing.

Results of Study 1 replicated findings of prior research demonstrating a main effect of smiling on attractiveness, in that smiling faces were rated as more attractive than faces displaying a neutral expression. ${ }^{6,7}$ These previous studies did not, however, address the influence of smiling as a function of attractiveness level, whereas that interaction was tested for and examined in Study 1.

A clear interaction pattern was established wherein the HA individuals were rated as higher in both attractiveness and happiness when smiling but the LA individuals were rated as higher only in happiness when smiling. Note that the lack of a difference in attractiveness ratings for the LA individuals as a function of emotional expression is in large part due to the high frequency of low attractiveness ratings (see Figure 2A) across participants. Thus, though individuals were included in the HA category if they were rated high in attractiveness for either emotional expression, the LA category only included individuals rated low in both emotional expressions. This restricted range effect is advantageous for Study 2, however, because as a result attractiveness varied based on emotionality in only the HA category. This difference in the response pattern, i.e., an effect of smiling for the LA category on happiness ratings but not on attractiveness ratings, is useful in drawing conclusions about the relative timing of the combination of attractiveness and emotionality in the brain. Namely, it helps answer the question of whether conveyed facial emotion needs to be processed before the physical emotional expression influences the brain response to attractiveness. 


\section{STUDY TWO}

Investigating the timing of the neural processing of attractiveness, emotionality, and their interaction can offer insights into how these factors are processed in the brain. In order to do so, it is crucial to know the relative timing of each of these three processes. Initial brain processing that differentiates faces from other visual stimuli has been shown to occur within $200 \mathrm{~ms}$ of stimulus presentation ${ }^{18}$ in parts of the left and right fusiform and inferior temporal gyri. With the use of scalp recordings, additional processing of the nature of the face, such as its relative attractiveness and emotional expression, has been found to occur after this and in different locations as detailed below.

Studies on the timing of the perception of attractiveness indicate that it is processed especially early. Werheid et al. discovered that an early posterior negativity (EPN; $\sim 250 \mathrm{~ms}$ ) occurs in addition to a late parietal positivity (400-600 ms) for attractive faces in relation to unattractive faces. ${ }^{9}$ Task specific effects, such as those resulting from explicitly judging relative attractiveness, can occur even earlier, with differential effects found for the P1 (100-140 ms) and N170 (140-190 ms) ERP components with larger magnitudes for attractive faces.9,20 Zhang and Deng also found an early N300 (230-330 ms) with greater negativity for attractive faces ${ }^{20}$ which we expect to be the early stage attractiveness response for passive viewing. We thus expected to observe an effect of attractiveness in the 230-300 $\mathrm{ms}$ time range during passive viewing.

Based on the literature, the timing of emotionality processing at first appears to be a bit more variable than that of attractiveness, but this is likely in part due to the various forms of emotion examined and the tasks required of participants. According to a recent review by Calvo and Nummenmaa, emotional expression is processed differently than neutral expression between 150 and $350 \mathrm{~ms}$, the range of N170 to EPN. ${ }^{21}$ The earliest differential responses are possibly based on negative emotions ${ }^{22}$ and/or task demands of making an explicit choice. ${ }^{17}$ Balconi and Pozzoli observed negative peaks around $230 \mathrm{~ms}$ elicited by faces displaying fear, anger, surprise, happiness, or sadness. ${ }^{22}$ Schupp et al. differentiated responses to threatening, friendly, and neutral facial expressions and found a greater and earlier overall effect for threatening stimuli. ${ }^{16}$ Furthermore, Calvo et al. found that for angry and fearful faces in relation to neutral ones, a differential response occurred between 175-250 ms and was present in the frontal and central scalp regions in addition to some posterior regions. ${ }^{10}$ They found that differential responses for happy faces in relation to neutral ones occurred later - around $330-430 \mathrm{~ms}$ - and were predominantly located in the posterior regions. ${ }^{10} \mathrm{We}$ thus expect to observe an effect of happy versus neutral expressions starting in the $300-400 \mathrm{~ms}$ time range.

Our review of the literature suggests that passive viewing of facial stimuli would result in an earlier brain response to attractiveness than emotional expression. To examine post-stimulus effects across categories, we used a component-independent experimental design which looks into the timing of effects specifically rather than parsing out the ERP components involved in advance. In An Introduction to the Event-related Potential Technique, Luck states this is the best strategy to use in order to avoid the obscurity typically enmeshed with determining which components compose an ERP waveform. ${ }^{33}$ Three possibilities exist for the timing of an interaction effect: (1) before the processing of emotion, (2) concurrent with the processing of emotion, or (3) after the processing of emotion. Respectively, these effects are estimated to occur approximately (1) less than $300 \mathrm{~ms}$ after the stimulus, (2) in the 300-400 ms time range, and (3) more than $400 \mathrm{~ms}$ after the stimulus. Early interaction effects occurring prior to explicit emotional processing (possibility 1) would suggest independent processing of attractiveness and emotional expression with emotional expression having a direct modulation on attractiveness processing. Late interaction effects occurring during or after both attractiveness and emotionality processing (possibilities 2 and 3) would suggest parallel or sequential dependent processing. As we are the first to our knowledge to look at these responses for the same set of stimuli in the same study, a specific hypothesis about the time at which an interaction would take place was not made.

\section{METHODS}

\section{Participants}

Data Set 1: For this study, 27 female students were recruited from the general student body. None were in Study 1. Students in psychology classes received course credit in exchange for their participation. No information about demographics or handedness was recorded. All participants had normal or corrected-to-normal vision. Anyone susceptible to seizures induced by flashing stimuli was not eligible to participate. The study was conducted in accordance with the guidelines of the Roanoke College Institutional Review Board, and all participants provided informed consent.

Data Set 2: For an unrelated study, ${ }^{24} 112$ students were recruited from the general student body. The data of 45 female students with a sufficient number of valid ERP trials (see below for more details) was also included in the current study. All other information given about participants in Data Set 1 is the same. 


\section{Equipment}

The equipment setup was the same as for other projects in the lab. ${ }^{25}$ A PowerLab 26T from AD Instruments was used to record the electroencephalography (EEG) signals. Five lead shielded electrodes transmitted signals from the participants' scalps to the PowerLab unit. The EEG signal was sampled at $400 \mathrm{~Hz}$ and underwent bandpass filtering between $0.5 \mathrm{~Hz}$ and $50 \mathrm{~Hz}$ with a Mains filter applied prior to recording. In order to reduce the possibility of artifacts, all trials were conducted in a dark room with minimal possible distractions. All software was run on a Dell XPS 15z laptop. Participants viewed stimuli on the laptop's internal 15" widescreen monitor. Stimuli were presented to participants using SuperLab 4.5 from Cedrus Corporation. An external 17" Dell monitor viewable only to experimenters presented the output of the EEG signals through LabChart 7 software from AD Instruments. The temporal presentation order of the stimuli was recorded using a StimTracker device from Cedrus Corporation.

\section{Stimuli}

Participants were presented with images of faces and of fingerprints. Fingerprints were included as a way to give participants the opportunity to blink during runs. The 80 face images were from the HA and LA categories of Study 1, i.e., they were of the 20 most attractive and the 20 least attractive individuals, each with two facial expressions. These images were 384 pixels high and 256 pixels wide, embedded in a gray rectangle $(\mathrm{RGB}=127)$. The 20 pictures of fingerprints were 383 pixels high and 254 pixels wide with no gray border. The fingerprints appeared as black lines $(\mathrm{RGB}=0)$ on a white background $(\mathrm{RGB}=255)$. All images were presented using SuperLab 4.5 on a gray background $(\mathrm{RGB}=130)$. At the beginning of each run, a white cross appeared in the center of the screen in order to guide fixation. The faces were centered on the same location as the middle of the cross, with the center of the screen being around the nose area of the face.

\section{Procedures}

Upon entering the experiment room, potential participants were prescreened and given an informed consent sheet. If they were susceptible to seizures, had metal in their heads, or did not have normal or corrected-to-normal vision, they were not eligible to participate $(n=0)$. Participants were additionally warned that the procedures of the study could possibly induce migraines and were asked to remove any jewelry on or around their face. After cleaning and abrading their forehead, the electrodes were put in place (see below for specific locations for each data set). Channel 1 focused on the anterior-posterior axis and included an electrode over the occipital lobe $(\mathrm{Oz})$ in order to detect general visual processing. Channel 2 included an electrode over the right temporal-parietal region (TP10) in order to detect initial face processing and capture the right-lateralized EPN. This effect has been localized to the right posterior region in prior studies.9,10,20 Though a small number of electrodes limits spatial resolution, the temporal resolution was sufficient to address our question of the relative timing of brain responses.

Participants were then asked to rest their chins on a pillow placed on top of a stack of books, and the lights were turned off. Stimuli were presented on a laptop screen approximately $30 \mathrm{~cm}$ from the participant. The LabChart 7 software was opened, and the experimenter evaluated the incoming signals to help ensure that the equipment was set up and functioning properly. Once the experimenter had tested the electrode connections for voltage consistently within the $+/-60 \mu \mathrm{V}$ range, five runs were conducted which lasted about three minutes each. Sometimes additional runs were conducted if equipment malfunctioned or participants experienced some sort of issue. For example, the SuperLab 4.5 software crashed on approximately $5 \%$ of the runs. In between runs, participants were always given the opportunity to rest their eyes if necessary. For each run, LabChart recorded the continuous EEG output from the PowerLab unit and the discrete timing of the stimulus presentations from the Stim'Tracker unit. The stimuli were presented to participants in a random order using SuperLab 4.5.

Each run consisted of the presentation of 100 trials, i.e., a single presentation of each of 80 face images and 20 fingerprint images. The task was simply to look at the face images while refraining from blinking and to blink when the fingerprint images appeared. At the beginning of each run, a white cross appeared in the center of the screen for $500 \mathrm{~ms}$. There was a $600 \mathrm{~ms}$ pause between when the cross was removed and when the first stimulus was presented. A comment line was inserted into the EEG data trace $100 \mathrm{~ms}$ prior to the presentation of each stimulus, with different comments specifying the different stimulus categories. Each stimulus image was presented for $300 \mathrm{~ms}$. Since judgements made based on viewing photographs for only $100 \mathrm{~ms}$ and without time constraints are highly correlated, ${ }^{26}$ a 300 ms presentation was sufficient for people to form an impression based on appearance. The interstimulus interval between the end of the presentation of one stimulus and the start of the next stimulus was 1300, 1400, or $1500 \mathrm{~ms}$. Each run took approximately three minutes. A large number of trials is needed to average out noise in the EEG signal, so the total number of runs was chosen to maximize the number of trials per stimulus that could be collected within a 30 min experimental time slot. After completion of the runs, participants were debriefed. The entire process took approximately 30 minutes per participant.

Data Set 1: The ground electrode was placed on the left side of the forehead approximately halfway between the eyebrows and hairline (FP1). The negative Channel 1 electrode was placed on the right side of the forehead halfway between the eyebrows and 
hairline (FP2), and the positive Channel 1 electrode was placed approximately an inch above the inion (OZ). The negative Channel 2 electrode was placed on the left earlobe (A1), and the positive Channel 2 electrode was placed about an inch behind and above the right ear (TP10). The electrode placed on A1 was disposable and was affixed to the earlobe with its adhesive backing. The other four electrodes were attached to the scalp using electrode paste and held in place with an elastic headband. Participants viewed the stimuli five times each. As there were 20 images per stimulus category, there were 100 trials per stimulus category across the five runs.

Data Set 2: The procedures were nearly identical to those of Data Set 1, though participants completed four runs rather than five. Additionally, for two out of the four runs, fingerprint images were not displayed, and participants were asked not to blink for the duration of the run (approximately 2.5 minutes). Also, there were slight differences in the placement of the electrodes. For Channel 1, the electrodes were all in the same anatomical regions but the sides of the forehead were reversed for the positive electrode and the ground electrode. Therefore, the data for Channel 1 is believed to be comparable to the other data set. For Channel 2, the negative electrode was moved from the earlobe to the left temple (F7) and the positive electrode remained in the same location (TP10). All five electrodes were attached to the scalp using electrode paste and held in place with elastic headbands. As there were 20 images per stimulus category, there were 80 trials per stimulus category across the four runs.

\section{Analysis}

Following data collection, the data were exported from LabChart 7 into a format that could be analyzed in a custom-designed MATLAB program for ERP analysis. The data were exported as a matrix containing the entire time course of the EEG recordings with a separate matrix indicating the corresponding temporal location and nature of the comments that specified which stimulus was presented when. As the shortest duration between successive stimuli was 1300 ms, the ERP for each trial was determined for a time span of $1200 \mathrm{~ms}$, starting $300 \mathrm{~ms}$ prior to the stimulus onset and ending $900 \mathrm{~ms}$ after the stimulus onset. The voltage values were scaled such that the baseline average, defined as the voltage amplitude from $300 \mathrm{~ms}$ to $100 \mathrm{~ms}$ prior to stimulus onset, was always 0 .

Artifacts were determined by whether or not the voltage within a trial ever reached an amplitude greater than $60 \mu \mathrm{V}$ above or below the baseline voltage, and a trial was discarded from further analysis if an artifact was present. Eye stability was not directly checked, though large eye movements or blinks would lead to artifacts in Channel 1 due to the location of an electrode on the frontalis muscle. Since artifacts in Channel 1 were associated with the loss of visual input for a given trial, corresponding trials in Channel 2 were also discarded when an artifact was present in Channel 1. Additional trials were discarded for Channel 2 alone if an artifact was present in only Channel 2. For Data Set 1, artifacts were present in $41 \%$ of trials across all participants, though the frequency of artifacts varied by participant from 1\% to $91 \%$. Participants who averaged less than 40 valid trials per condition in Channel 1 were eliminated from further analysis. The data of four participants was discarded, leaving 23 remaining participants. For the remaining participants, an average of $35 \%$ of trials had artifacts, i.e., $65 \%$ were considered valid trials. For Data Set 2 , participants were only included from the beginning if they averaged at least 40 valid trials per condition in Channel 1 and individuals varied from $58 \%$ to $97 \%$. An average of $26 \%$ of trials had artifacts, i.e., $74 \%$ were considered valid trials in Channel 1.

ERPs were determined separately for the four conditions (HA smiling, HA neutral, LA smiling, LA neutral) by averaging across all valid trials. The magnitude of the response for a particular category was the average voltage across time within a particular time window that could be used to examine main effects of attractiveness and emotionality as well as their interaction. Consistency of the effects across participants for each time window was determined by a 2x2 RM ANOVA with attractiveness category and emotional expression as the factors. RM ANOVAs were performed within $100 \mathrm{~ms}$ time windows using a sliding window procedure that shifted the analysis window by $10 \mathrm{~ms}$ for each subsequent analysis. Significance within a time window is reported based on the midpoint of the time window, i.e., a midpoint of $190 \mathrm{~ms}$ refers to a window of 140-240 ms. The $p$-values for the main effects and interaction effect were recorded within each time window so that subsequent analysis could be performed on when in time each effect was present. A nominal significance level of $p<.05$ was used. Caution needs to be used, however, in interpreting significance if the range of time windows that are significant is small, i.e., less than three consecutive windows, due to the high number of statistical tests being run on each time course and the substantial overlap of adjacent time windows.

\section{RESULTS}

Valid Trials

Not all participants ended up with the same number of valid trials because individual trials with artifacts were discarded from the data. It was possible that the number of valid trials varied with the conditions and, furthermore, behavioral artifacts could have been differentially caused by the conditions. In order to test this, a 3-way RM ANOVA was run for each data set with the attractiveness category (HA vs. LA), emotional expression (smile vs. neutral expression), and channel (Channel 1, including the frontalis muscle, vs. Channel 2, including the temporalis muscle) as factors. 
Data Set 1: There was a main effect of channel, $F(1,22)=5.2, p=.032, \eta^{2}=.047$, with more valid trials in Channel $1(66.7 \%)$ than Channel $2(63.5 \%)$ and also of emotion, $F(1,22)=10.6, p=.004, \eta^{2}=.122$, with more valid trials for neutral $(67.7 \%)$ than smiling $(62.5 \%)$ expressions (Figure $4 \mathrm{~A})$. The interaction effect between channel and emotionality was also significant, $F(1,22)=$ $5.2, p=.032, \eta^{2}=.047$, as the frequency of valid trials for the smiling expression dropped from $65.7 \%$ to $59.2 \%$ when artifacts specific to Channel 2 were included but no artifacts were found specific to Channel 2 for the neutral expression. Neither the main effect of attractiveness category $(F<<1)$ nor any of the interaction effects associated with attractiveness category $(F$ s $<<1)$ were significant. Therefore, subsequent analyses looking at the timing of interaction effects in the ERPs within Channel 1 involving attractiveness can be considered unbiased with regards to the number of valid trials.

Data Set 2: Four participants were excluded from this analysis because they did not have any valid trials in the smiling conditions for Channel 2, though all results were qualitatively similar when they were included. There was a main effect of channel, $F(1,40)=$ $15.1, p<.001, \eta^{2}=.077$, with more valid trials in Channel $1(74.4 \%)$ than Channel $2(69.2 \%)$ and also of emotion, $F(1,40)=6.4, p$ $=.016, \eta^{2}=.044$, with more valid trials for neutral $(73.8 \%)$ than smiling $(69.8 \%)$ expressions (Figure 4B). The interaction effect between channel and emotionality was also significant, $F(1,40)=15.1, p<.001, \eta^{2}=.077$, as the frequency of valid trials for the smiling expression dropped from $75.0 \%$ to $64.6 \%$ when artifacts specific to Channel 2 were included but no artifacts were found specific to Channel 2 for the neutral expression. Neither the main effect of attractiveness category $(F<<1)$ nor any of the interaction effects associated with attractiveness category $\left(F^{\prime}<<1\right)$ were significant.
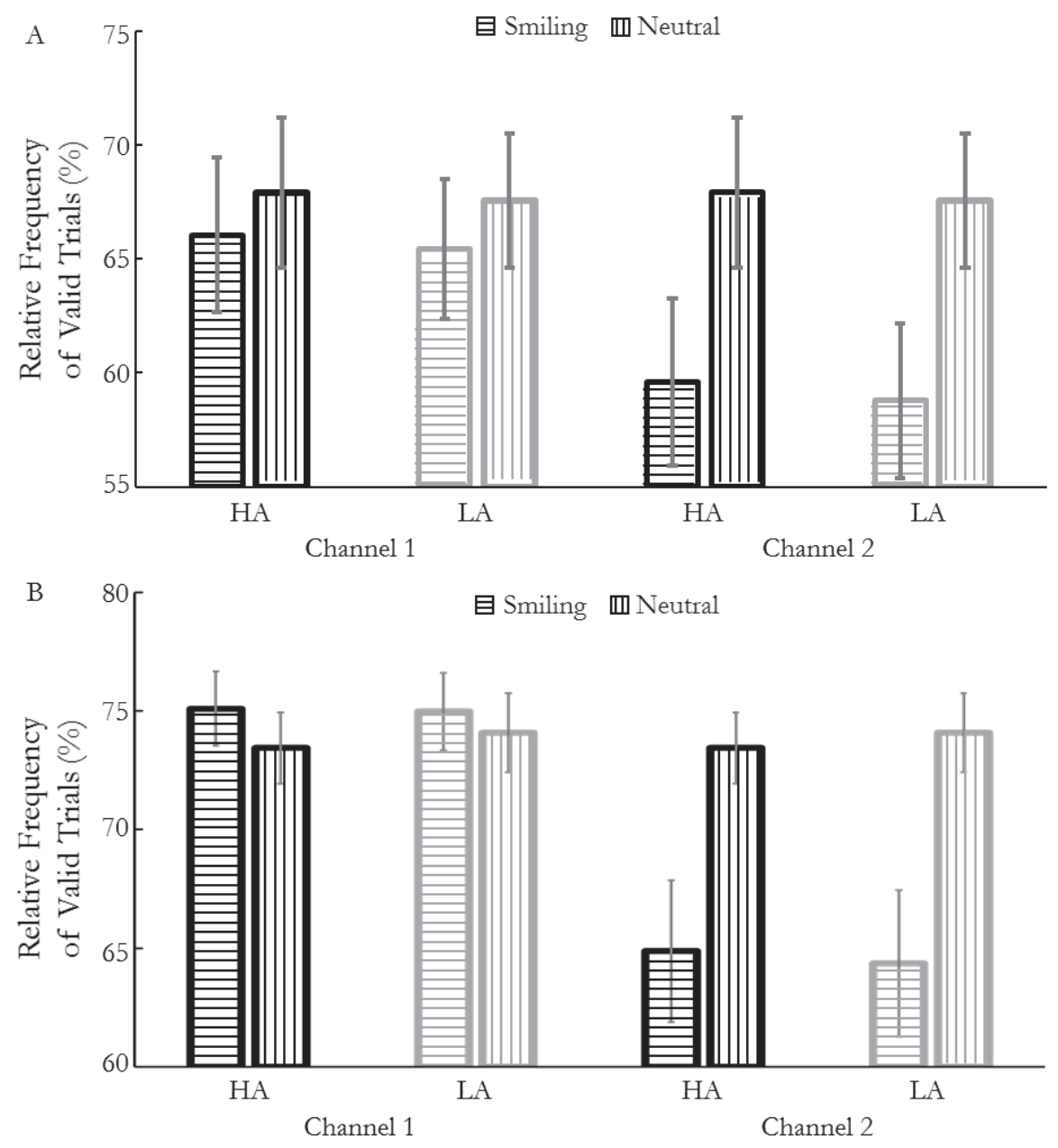

Figure 4. The percentage of trials for each of the Study 2 conditions averaged across participants, graphed separately by channel. Note that all trials excluded in Channel 1 were also excluded in Channel 2 but trials could be excluded in Channel 2 without being excluded in Channel 1. Error bars show 1 standard error of the mean. A) Results from Data Set 1. B) Results from Data Set 2. 


\section{Main Effects and Interaction Effects in ERP Data}

The time course for the ERPs as a function of attractiveness category and emotional expression within Channel 1 is shown in Figure 5A for Data Set 1 and Figure 5B for Data Set 2. Of particular interest is the differential timing of when brain responses appear for the main effects of attractiveness and emotionality as well as the interaction effect between them. The nature of the observed interaction effect, i.e., whether the brain responses more closely resemble the observed interaction patterns in Study 1 based on attractiveness or on happiness ratings, is also of interest.

Data Set 1: A significant main effect of attractiveness with a more positive voltage for HA faces was found for time windows of midpoints $190 \mathrm{~ms}$ to $310 \mathrm{~ms}$. A later main effect of attractiveness with a more negative voltage for HA faces was found from midpoints $730 \mathrm{~ms}$ to $780 \mathrm{~ms}$. No post-stimulus time windows exhibited a significant main effect at the level of $p<.05$ for emotional expression, though a marginally significant main effect of emotional expression at the level of $p<.075$ was found for time windows of midpoints $300 \mathrm{~ms}$ to $340 \mathrm{~ms}$. A significant interaction effect was seen for just a time window midpoint of 210 ms. Within that window, there was a difference between HA smiling faces and HA neutral faces, $t(22)=2.7, p=.012$, but not between LA smiling faces and LA neutral faces, $t(22)=0.2, p=.869$. This is the same pattern of interaction as was found for the attractiveness ratings in Study 1. There were no significant main effects or interaction effects in Channel 2 (all $p$ 's $>.12$ ).

Data Set 2: A significant main effect of attractiveness with a more positive voltage for HA faces was found for time windows of midpoints $200 \mathrm{~ms}$ to $330 \mathrm{~ms}$. A significant main effect of emotionality with a more positive voltage for neutral faces was found for time windows of midpoints $360 \mathrm{~ms}$ to $490 \mathrm{~ms}$. No post-stimulus time windows exhibited a significant interaction effect at the level of $p<.05$. Even for the specific time point where an interaction effect was observed in Data Set 1, the interaction effect was not close to significant $(p=.40)$. For Channel 2, the data for individual participants was too unreliable to analyze.
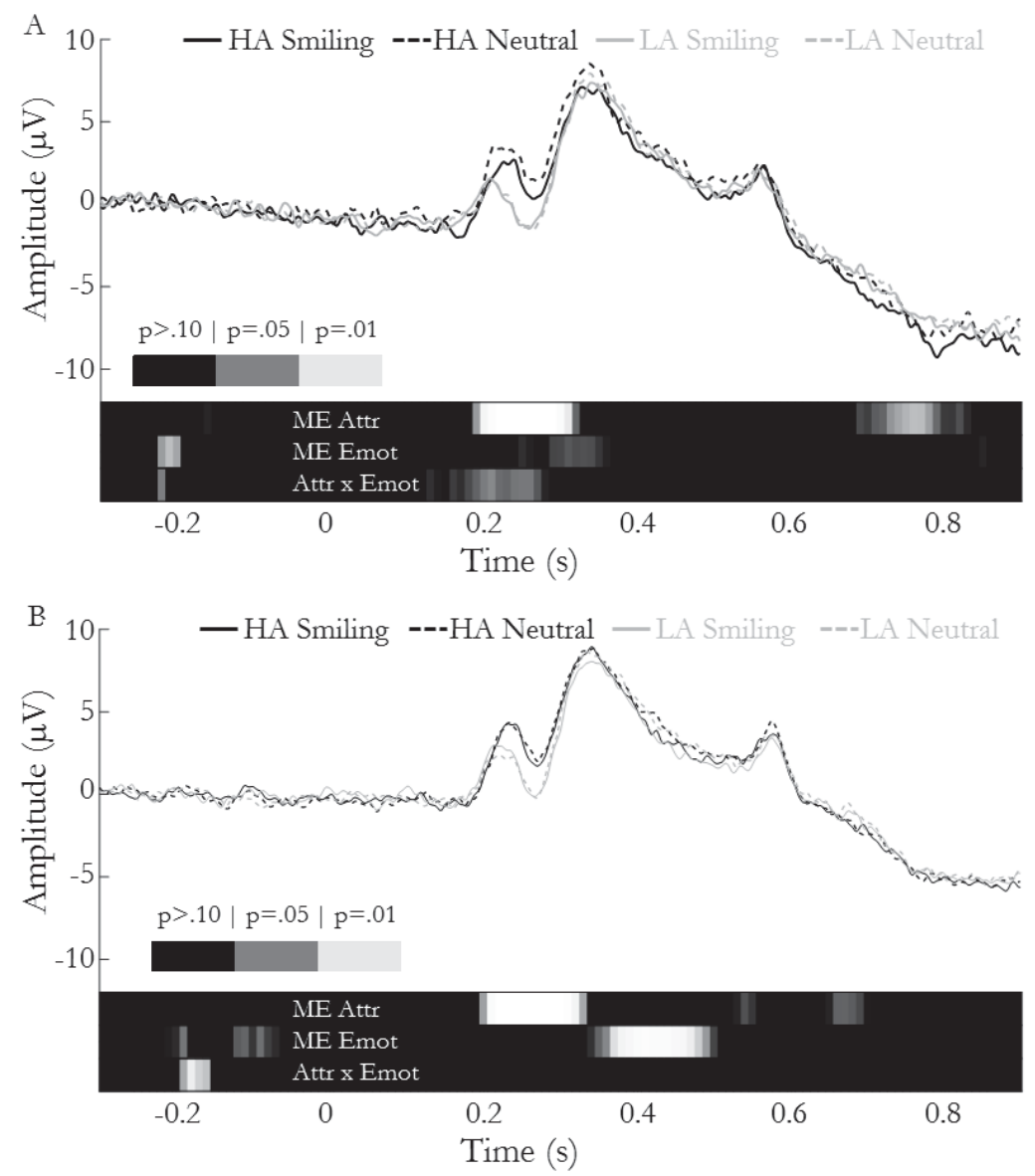

Figure 5. The ERP signals for each of the Study 2 conditions is shown across time. The output of RM ANOVAs run within various 100 ms analysis windows is also shown with the $p$-values reflected in the shading of the rectangles. The middle of the shaded rectangle is the midpoint of the 100 ms analysis window. Results are shown for Channel 1 only as Channel 2 data were not statistically significant. The stimulus onset was at time $=0 \mathrm{~s}$. A) Results from Data Set 1 . B) Results from Data Set 2. 


\section{DISCUSSION}

The relative timing and magnitude of ERPs specific to facial attractiveness and emotional expression were observed across two sets of data using independent sets of participants but similar data methods, including identical stimuli. Consistent with prior research, ${ }^{9,20}$ a main effect of attractiveness level was found roughly 200-300 ms after the stimulus. Also consistent with prior research, ${ }^{10}$ a main effect of smiling versus neutral expression was observed subsequent to this, roughly $300-500 \mathrm{~ms}$ after the stimulus. Data Set 1 suggests that the established pattern of attractiveness ratings of HA and LA face stimuli in Study 1 may correspond to observable patterns of brain responses. The same interaction effect was observed in both Study 1 attractiveness ratings and Data Set 1 of Study 2, even though the studies had separate sets of participants and Study 1 involved explicit ratings of the faces while in Study 2 participants were not required to make explicit ratings of the stimuli but rather viewed them passively. The observed interaction pattern of early brain responses was consistent with those observed for attractiveness ratings but not for happiness ratings. Caution is required in drawing conclusions about the neural processing of attractiveness and emotionality based on this interaction effect, however, because it occurred at only a single analysis time point and failed to replicate in Data Set 2 with a larger number of participants.

It is important to note that while our observed timing of the attractiveness and emotionality ERPs was consistent with previous research, the direction of the magnitudes of the effects was inconsistent. That is, previous findings on the early attractiveness response, e.g., EPN, have tended to find that attractive faces have a more negative amplitude than unattractive faces $9,15,27$ whereas we found a more positive response for attractive faces. Similarly, happy expressions consistently show a more negative amplitude than neutral expressions ${ }^{17,18,20}$ whereas we found a more positive response for smiling versus neutral faces. This consistent inversion of the direction of the observed effects can be explained by the fact that we used a bipolar EEG setup and our EEG channel 1 was defined with the posterior lead, Oz, as the positive input versus the anterior lead, FP1/2, as the negative input. Most other EEG setups assess each location roughly independently in relation to a general or grand mean measure. Therefore, we still believe that our results show patterns of brain response for main effects of attractiveness and emotionality consistent with the published literature.

A novel finding in addition to that of the potential timing of the interaction effect was that the smiling conditions were associated with participants having fewer valid trials. Based on the observations made by researchers during data collection, it is suspected this resulted from participant facial responses such as smiling, as well as from laughter. This is supported by the fact that Channel 2 - which would have been more likely to pick up on mouth movements through the temporalis muscle - had artifacts beyond those in Channel 1 only for the smiling conditions. Previous research has found differential facial expressions in viewers wherein more negative expressions are made when viewing unattractive faces. ${ }^{28}$ Our results are better explained by the chameleon effect whereby people tend to unconsciously mimic the expressions of others ${ }^{29}$ - as we observed more smiling behaviors in participants viewing happy expressions regardless of attractiveness level. The different levels of artifacts do not account for the observed interaction effect in brain responses though because the interaction effect found in ratings and brain responses was specific to different levels of attractiveness and the chameleon effect is only related to emotional expression.

\section{GENERAL DISCUSSION}

The current studies investigated the effects of attractiveness and smiling through behavioral and electrophysiological methods. In Study 1, self-report ratings were used to establish sets of high attractive (HA) and low attractive (LA) faces based on average participant responses and to determine a differential interaction effect of attractiveness and emotional expression based on attractiveness and happiness ratings. A difference in attractiveness ratings was found between smiling and neutral HA faces but not between smiling and neutral LA faces, whereas a difference in happiness ratings as a function of emotional expression was present for both the HA and LA categories but was of different magnitudes. Study 2 found that attractiveness categories were differentiated in the ERP prior to the differentiation based on emotional expression. The number of artifacts in Channel 2 varied with emotional expression, irrespective of attractiveness level. This was likely due to participants' mimicry of the smiling expressions as a result of the chameleon effect ${ }^{29}$ and thus seemed to be reflective of a behavioral response lacking an interaction effect. The interaction effect of attractiveness and emotional expression, when observed, overlapped with the early attractiveness response and occurred before the emotionality-based response. Furthermore, a difference was found between the smiling and neutral images for HA individuals but not LA individuals, indicating that the observed ERP interaction effect was consistent with the self-report ratings of attractiveness but not of happiness. While such a set of findings supports an overall conclusion that smiles have a direct and initial impact on the perception of individuals' relative attractiveness prior to emotionality being explicitly processed, suggesting independent brain processing, the failure to replicate the neural interaction effect leaves open the possibility for parallel or sequential dependent processing with an interaction effect that takes place concurrent with or following the brain processing of emotional expression. 
The establishment of four subsets of faces (HA smiling, LA smiling, HA neutral, and LA neutral) may be applicable in many studies of psychology with particular benefits if the same set of stimuli are used repeatedly across studies. For example, the subset may be used to test the chances of being hired based upon a mock employee resume and an included photo from the given subset in an industrial-organizational study. Further studies examining attractiveness with a similar approach could focus on establishing a subset of female faces for each condition while controlling for age-related effects. The FERET database ${ }^{19}$ may be ideal for this purpose based on the large number of faces included in it and the fact that it is provided for free use in scientific research. An alternative could be the establishment of a stimulus repository so that various studies are able to use the same set of face images in order to increase consistency amongst research studies and to make replication easier.

In assessing the findings of these studies, it is important to note their limitations. First, female ratings of male faces were used, and therefore findings may not generalize to male ratings of female faces, especially considering the differences in how men and women perceive facial attractiveness and smiling. While happiness is the most attractive emotional expression in females, happiness is the least attractive male expression, and pride, the least attractive female expression, is the most attractive male expression. ${ }^{30}$ An evolutionary hypothesis frames sex differences in the attractiveness of emotional expressions as due to a difference in mating strategies wherein women seeking long-term partners find smiling faces more attractive but women seeking short-term partners prefer masculine faces over smiling faces. ${ }^{13}$ Therefore, we could surmise that part of our observed variance in ratings may have resulted from differences in participants' internalized mating strategies. Another consideration is that there may be less difference in the facial expressiveness of high and low attractive men compared to high and low attractive women, ${ }^{14}$ further indicating that results may be different if males rate images of females.

Second, in Study 1, some participants rated some individuals as happier when displaying a neutral expression as compared to a happy expression. One individual was rated in such a manner overall. We created the HA and LA categories based solely on average attractiveness ratings. Happiness ratings were examined in a global sense to verify that smiling images were rated as happier than images with a neutral expression, but we did not look at relative happiness ratings for individual images until after running Study 2. It would likely be better for future studies using these same categories to exclude images in the LA category rated as less happy when smiling and possibly even any rated as similarly happy when smiling. More generally, future studies would benefit from checking that ratings of stimuli are consistent with what they should be on an individual basis in addition to on a global basis and from subsequently eliminating stimuli from analysis that do not pass this methodological check.

In addition, a substantial portion of the images were of individuals who were 20-40 years older than the participants. Images of older individuals were included in the image set to allow for broader conclusions about patterns in the perception of attractiveness and emotionality. This was beneficial in examining differences in the overall ratings of depicted individuals in Study 1 and thus in the discovery of the interaction effects in the ratings. However, it proved to be a confound in later analyses when the individuals were divided into groups based on their attractiveness levels, as older individuals tended to be rated as less attractive than younger individuals. Therefore, attractiveness was not the only characteristic that varied between the LA, MA, and HA groups, restricting our ability to draw conclusions. Since the raters were college-aged females, it was not surprising that images of older individuals were generally judged as less attractive. If the raters had been older, they may have found the older individuals more attractive than the participants used in the current studies, though there is evidence for a clear youth bias in attractiveness regardless of the age of the rater. ${ }^{31}$ Furthermore, there was less ethnic diversity in the HA category than the LA category after sorting. This could have come about due to either a relatively homogenous set of participants or a discrepancy in the range of ethnicity in the images as a function of age. In future studies on the effects of attractiveness and emotional expression, age-related confounds could be avoided by using a wider range of images of individuals who are all of a similar age to that of the participants.

Overall, participants seemed to smile or laugh more while viewing smiling images than while viewing neutral images based on an increase in artifacts. Anecdotally, certain images within the smiling and neutral categories seemed to be associated with a high number of artifacts, but only the category of stimuli, not the individual image being displayed at a particular point, was recorded. Therefore, we are unable to determine whether participants reacted differently to individual stimuli, i.e., if particular images made participants laugh or smile, leading to an abundance of artifacts. Future studies may wish to record the presentation order of all individual stimuli so that differences in reactions to individual images can be analyzed.

Processing of attractiveness was expected to occur earlier than processing of emotion, but it was not previously clear whether emotional expression needed to be processed before it could influence perceived attractiveness. Our results partially imply modular neuroarchitecture for the neural processing of face qualities, ${ }^{11}$ wherein explicit processing of emotionality is not required to occur before an interaction of attractiveness and emotional expression. Study 1 also suggested the existence of an interaction between attractiveness and perceived happiness levels, but we are not able to draw conclusions about the existence or timing of that interaction on a neural processing level due to the placement of our electrodes. A location such as Pz may have spoken to a 
happiness-based interaction, as suggested by ERPs resulting from emotional stimuli centered on a more parieto-central location (see Figure 2 of Calvo et al.18; Figure 1 of Schupp et al.16). Further research with electrodes at a variety of locations could help to fully clarify the nature of the neurological combination of facial attractiveness and emotional expression.

\section{REFERENCES}

1. Sunnafrank, M., and Ramirez Jr., A. (2004). At first sight: Persistent relational effects of get-acquainted conversations. Journal of Social and Personal Relationships, 21, 361-379. doi:10.1177/0265407504042837

2. Lennon, S. (1988). Physical attractiveness, age, and body type. Home Economics Research Journal, 16, 195-203. doi: $10.1177 / 1077727$ X8801600304

3. Dion, K., Berscheid, E., and Walster, E. (1972). What is beautiful is good. Journal of Personality and Social Psychology, 24, 285-290. http://dx.doi.org/10.1037/h0033731

4. Langlois, J. H., Kalakanis, L., Rubenstein, A. J., Larson, A., Hallam, M., and Smoot, M. (2000). Maxims or myths of beauty? A meta-analytic and theoretical review. Psychological Bulletin, 126, 390-423. doi:10.1037/0033-2909.126.3.390

5. Dimberg, U. and Thunberg, M. (1998). Rapid facial reactions to emotional facial expressions. Scandinavian Journal of Psychology, 39, 39-45. doi: 10.1111/1467-9450.00054

6. Lau, S. (1982). The effect of smiling on person perception. The Journal of Social Psychology, 117, 63-67. doi:10.1080/00224545.1982.9713408

7. Abel, M.H., and Watters, H. (2005). Attributions of guilt and punishment as functions of physical attractiveness and smiling. The Journal of Social Psychology, 145, 687-702. doi:10.3200/socp.145.6.687-703

8. O’Doherty, J., Winston, J., Critchley, H., Perrett, D., Burt, D.M., and Dolan, R. J. (2003). Beauty in a smile: the role of medial orbitofrontal cortex in facial attractiveness. Neuropsychologia, 41, 147-155. doi: 10.1016/s0028-3932(02)00145-8

9. Werheid, K., Schacht, A., and Sommer, W. (2007). Facial attractiveness modulates early and late event-related brain potentials. Biological Psychology, 76, 100-108. doi:10.1016/j.biopsycho.2007.06.008

10. Calvo, M. G., Marrero, H., and Beltrán, D. (2013). When does the brain distinguish between genuine and ambiguous smiles? An ERP study. Brain and Cognition, 81, 237-246. doi:10.1016/j.bandc.2012.10.009

11. Bzdok, D, Langner, R., Hoffstaedter, F., Turetsky, B. I., Zilles, K., and Eickhoff, S. B. (2011). The modular neuroarchitecture of social judgements on faces. Oxford University Press, 22, 951-961. doi:10.1093/cercor/bhr166.

12. Rolls, E.T. (2000). Precis of the brain and emotion. Behavioral and Brain Sciences, 23, 177-234. doi: $10.1017 / \mathrm{s} 0140525 \mathrm{x} 00002429$

13. Okubo, M., Ishikawa, K., Kobayashi, A., Laeng, B., and Tommasi, L. (2015). Cool guys and warm husbands: The effect of smiling on male facial attractiveness for short- and long-term relationships. Evolutionary Psychology, 1-8. doi:10.1177/1474704915600567

14. Rennels, J. L., and Kayl, A. J. (2015). Differences in expressivity based on attractiveness: Target and perceiver effects? Journal of Experimental Social Psychology, 60, 163-172. doi:10.1016/j.jesp.2015.05.012

15. Chen, J., Zhong, J., Zhang, Y., Li, P., Zhang, A., Tan, Q., and Li, H. (2012). Electrophysiological correlates of processing facial attractiveness and its influence on cooperative behavior. Neuroscience Letters, 517, 65-70. doi:10.1016/j.neulet.2012.02.082

16. Schupp, H. T., Öhman, A., Junghöfer, M., Weike, A. I., Stockburger, J., and Hamm, A. O. (2004). The facilitated processing of threatening faces: An ERP analysis. Emotion, 4, 189-200. doi:10.1037/1528-3542.4.2.189

17. Rellecke, J., Sommer, W., and Schacht, A. (2012). Does processing of emotional facial expressions depend on intention? Time-resolved evidence from event-related brain potentials. Biological Psychology, 90, 23-32. doi:10.1016/j.biopsycho.2012.02.002

18. Calvo, M. G., and Beltrán, D. (2013). Recognition advantage of happy faces: Tracing the neurocognitive processes. Neuropsychologia, 51, 2051-2060. doi:10.1016/j.neuropsychologia.2013.07.010

19. Phillips, P. J., Raus, P. J., and Der, S. Z. (1996). FERET (face recognition technology) recognition algorithm development and test results. Army Research Laboratory.

20. Zhang, Z., and Deng, Z. (2012). Gender, facial attractiveness, and early and late event-related potential components. Journal of Integrative Neuroscience, 11, 477-487. doi:10.1142/s0219635212500306

21. Calvo, M. G., and Nummenmaa, L. (2015). Perceptual and affective mechanisms in facial expression recognition: An integrative review. Cognition and Emotion, 1-26. doi:10.1080/02699931.2015.1049124

22. Balconi, M. and Pozzoli, U. (2003). Face-selective processing and the effect of pleasant and unpleasant emotional expressions on ERP correlates. International Journal of Psychophysiology, 49, 67-74.

23. Luck, S. J. (2005). An introduction to the event-related potential technique. Cambridge, MA: MIT Press.

24. Shields, S. M., Morse, C. E., and Nichols, D. F. (2015, March). An exploration on the reduction of artifacts in EEG studies. Poster presented at the annual SYNAPSE undergraduate neuroscience conference, Asheville, NC. 
25. Hurless, N., Mekic, A., Pena, S., Humphries, E., Gentry, H., and Nichols, D. F. (2013). Music genre preference and tempo alter alpha and beta waves in human non-musicians. Impulse, 1-11.

26. Willis, J., and Todorov, A. (2006). First impressions: Making up your mind after a 100-ms exposure to a face. Psychological Science, 17, 592-598. doi:10.1111/j.1467-9280.2006.01750.x

27. Allison, T., Ginter, H., McCarthy, G., Nobre, A. C., Puce, A., Luby, M., and Spencer, D. D. (1994). Face recognition in human extrastriate cortex. Journal of Neuropsychology, 71, 821-825.

28. Principe, C. P., and Langlois, J. H. (2011). Faces differing in attractiveness elicit corresponding affective responses. Cognition and Emotion, 25, 140-148. doi:10.1080/02699931003612098

29. Chartrand, T. L., and Bargh, J. A. (1999). The chameleon effect: The perception-behavior link and social interaction. Journal of Personality and Social Psychology, 76, 893-910. doi:10.1037/0022-3514.76.6.893

30. Tracy, J. L., and Beall, A. T. (2011). Happy guys finish last: The impact of emotion expressions on sexual attraction. Emotion, 11, 1379-1387. doi:10.1037/a0022902

31. McLellan, B., and McKelvie, S. J. (1993). Effects of age and gender on perceived facial attractiveness. Canadian Journal of Behavioural Science, 25, 135-142. doi:10.1037/h0078790

\section{ABOUT THE STUDENT AUTHORS}

Paige Arrington graduated in May 2012, after the completion of Study 1. Stephanie Shields and Caitlin Morse graduated in May 2017.

\section{PRESS SUMMARY}

Prior research has shown that attractiveness and emotionality affect perception independently and interact to influence how a person perceives others, though the timing of the neural processing of this interaction is unknown. The present studies aimed to investigate this interaction and how it occurs through behavioral, specifically self-report, and physiological, specifically electrophysiological, methods using faces that varied in both attractiveness and emotional expression. Faces of high attractiveness were rated higher by participants on happiness in addition to attractiveness when smiling, but no effect of emotional expression on attractiveness ratings was found for faces of low attractiveness. A neurological interaction effect was observed in one data set coinciding with a main effect of attractiveness and prior to a main effect of emotional expression, suggesting independent processing of attractiveness and emotion. However, a neurological interaction effect was not seen in a second data set, though evidence was found consistent with modular processing of attractiveness and emotional expression. Further research is necessary to clarify the nature of the neurological combination of facial attractiveness and emotional expression. 